\title{
Effectiveness of Combined Yoga and Psychological Intervention on Cancer-Related Fatigue in Cervical Cancer Chemotherapy Patients
}

\author{
Hang Yang ${ }^{1, *}$, Qian Zhang ${ }^{1}$, Lixiu Liu ${ }^{2}$ \\ ${ }^{1}$ Shaanxi University of Chinese Medicine, Xianyang, China. \\ ${ }^{2}$ Affiliated Hospital of Shaanxi University of Chinese Medicine, Xianyang, China.
}

How to cite this paper: Hang Yang, Qian Zhang, Lixiu Liu. (2021) Effectiveness of Combined Yoga and Psychological Intervention on Cancer-Related Fatigue in Cervical Cancer Chemotherapy Patients. International Journal of Clinical and Experimental Medicine Research, 5(1), 19-24.

DOI: 10.26855/ijcemr.2021.01.005

Received: December 7, 2020

Accepted: January 3, 2021

Published: January 13, 2021

*Corresponding author: Hang Yang, Shaanxi University of Chinese Medicine, Xianyang, China.

Email: 654394368@qq.com

\begin{abstract}
Objective: To investigate the effect of yoga and psychological intervention on cancer-related fatigue in cervical cancer patients undergoing chemotherapy. Methods: Eighty-three cervical cancer patients undergoing chemotherapy were divided into a control group $(n=38)$ and an observation group $(n=45)$ according to the order of admission. The results of quality of life scale, anxiety and depression scores of the 2nd, 4th and 6th chemotherapy sessions after the intervention. Results: The total fatigue during and after chemotherapy was higher in the observation group than in the control group $(\mathrm{P}<0.05)$; the SF-36 Brief Quality of Life Scale scores of patients in the observation group were better than those of the control group after the intervention, and the difference was statistically significant; the mental health patents of the observation group were significantly better than those of the control group after the intervention $(\mathrm{P}<0.05)$; the anxiety and depression scores of patients in the two groups were not statistically significant $(\mathrm{P}>0.05)$; the anxiety and depression scores of patients in the two groups were compared, and the difference was not statistically significant $(\mathrm{P}>0.05)$. The anxiety and significance scores of the observation group after the 4th and 6th chemotherapy were significantly lower than those of the 2nd chemotherapy and lower than those of the control group $(\mathrm{P}<0.05)$. Conclusion: The combination of yoga and psychological intervention in cervical cancer patients during chemotherapy can relieve cancer-induced fatigue, improve patients' quality of life, and contribute to disease recovery.
\end{abstract}

\section{Keywords}

Cervical Cancer, Carcinogenic Fatigue, Yoga, Psychological Interventions

\section{Introduction}

Cervical cancer is one of the most common malignant tumors in women worldwide. Treatment is mainly surgery and radiotherapy, supplemented by chemotherapy [1]. Some studies have shown that $60 \%-90 \%$ of patients receiving anticancer therapy complain of fatigue [2], which is characterized by a series of discomfort symptoms such as fatigue, laziness, and dysfunction. According to the National Comprehensive Cancer Network (NCCN), carcinogenic fatigue is a subjective, painful, and persistent fatigue caused by cancer or cancer treatment that is unrelated to recent activities and significantly affects the patient's physical function [3]. Severe cancer-induced fatigue can 
cause patients to discontinue treatment, affect the effectiveness of treatment, and seriously affect patients' quality of life [3]. The purpose of this article is to investigate the effect of yoga resistance training combined with psychological intervention on cervical cancer patients' carcinogenic fatigue and quality of life. 83 cervical cancer patients from the Gynecologic Cancer Center, Affiliated Hospital of Shaanxi University of Traditional Chinese Medicine were selected for the intervention.

\section{Object and methods}

\subsection{Object of Study}

Eighty-three patients with cervical cancer who received postoperative chemotherapy at the Gynecologic Oncology Center of the Affiliated Hospital of Shaanxi University of Traditional Chinese Medicine from October 2019 to October 2020 were enrolled in the study. Inclusion criteria: (1) cervical cancer diagnosed by pathology; (2) no communication disorders; (3) no combination of serious chronic diseases such as respiratory, circulatory and digestive diseases; (4) voluntary participation in the study and signing of informed consent. Exclusion criteria: (1) patients with concomitant other cancers or serious chronic diseases; (2) patients who could not adapt to resistance training; (3) patients who withdrew from the study. The 83 patients were divided into a control group ( $\mathrm{n}=38$ ) and an observation group ( $\mathrm{n}=45)$ according to the order of admission. The control group was 20 to 80 years old, with a mean age of 48.67 .2 years; 30 patients were married, 5 were unmarried, and 3 were divorced. In the observation group, the age ranged from 22 to 78 years, with a mean age of 48.26 .9 years; 34 patients were married, 6 were unmarried, and 5 were divorced. There was no statistically significant difference $(\mathrm{P}>0.05)$ between the two groups in terms of age and marital status, so the two groups were comparable. This study was approved by the ethics committee of our hospital.

\subsection{Methods}

\subsubsection{Intervention Methods}

The control group adopted the following conventional intervention methods: (1) Health education: Through pictures and videos, health education was conducted to deepen patients' understanding of cervical cancer and the causes and clinical manifestations of cancer-induced fatigue, with emphasis on the prevention of cancer-induced fatigue and measures to cope with it in chemotherapy patients. By strengthening health education, we aim to establish patients' correct knowledge of the disease, thus building their confidence in treatment and improving their cooperation. At the same time, it also actively mobilizes patients' family members to be more caring, compassionate and understanding, so as to alleviate patients' worries about the burden of the disease on them. (2) Exercise care: Advise patients to pay attention to rest and reduce the amount of activity. (3) Dietary care: the dietary principle of chemotherapy patients is mainly high protein, easy to digest and light diet, telling patients to choose foods high in vitamin and protein, and to properly intake fresh vegetables and fruits, and avoid fried and spicy foods.

The observation group was given yoga resistance training and psychological intervention on the basis of the control group (in addition to the exercise care of the control group).

(1) Yoga impedance training intervention, as follows: (1) firstly, adjust breathing: guide patients to adjust breathing rhythm for $5 \mathrm{~min}$; (2) yoga practice session: play soothing music, the exercise mainly involves the movement of the body and limbs, slow down the speed during the initial exercise, gradually increase the intensity of the exercise, each exercise for $30 \mathrm{~min}$; (3) resting phase, patients take supine position. In the posture, keep your feet open and at the same distance from your shoulders, relax your body and breathe to music. Starting from the first chemotherapy treatment, group yoga practices 4 times a week until the end of the chemotherapy cycle.

(2) Psychological care: Conduct a comprehensive assessment of the patient's psychological state in order to understand the patient's psychological state. Patiently listen to the patient to understand the feelings in the patient's heart, grasp the patient's emotional changes, and accurately assess the patient's psychological state. Psychological interventions are given by psychologists and administered during each hospitalization for chemotherapy until the end of the chemotherapy cycle.

\subsubsection{Data collection}

A questionnaire was used to investigate. (1) The Cancer-relate fatigue (CRF) Scale [4], which is a 25-item survey of cancer fatigue, consists of three dimensions: physical fatigue, emotional fatigue, and cognitive fatigue, and is scored on a Likert 5 scale with a range of 1 to 5 points, with higher scores indicating more severe fatigue. (2) The SF-36 Brief Quality of Life Scale [5]: includes 8 dimensions of mental health, emotional functioning, social functioning, vitality, general health, physical health, physical functioning, and physiological functioning, and each di- 
mension has a full score of 100 points. The higher the score, the higher the quality of life. (3) Anxiety and depression were assessed by the Anxiety Inventory and Depression Inventory respectively [6], with higher scores indicating more significant depression and anxiety. (4) Psychological Status Test [7] criteria: There are 30 items in total, including social-behavioral problems (10 items), personal emotional problems (8 items), habitual problems (6 items), and study and work problems (6 items), with each item scoring 1 to 3 points.

\subsubsection{Evaluation Criteria}

We compared the changes of CRF before, during (2nd and 4th chemotherapy) and after (end of 6th chemotherapy) chemotherapy, and the changes of SF-36 Brief Quality of Life Scale, depression and anxiety scores, and mental health status scores before and after the intervention in these two groups.

\subsubsection{Statistical Analysis}

Excel and SPSS22.0 software were used for data entry and statistical analysis, and t-tests were performed for the measures. Statistical significance was indicated by $\mathrm{P}<0.05$.

\section{Results}

\section{Comparison of CRF between two groups of patients during chemotherapy}

There was no statistically significant difference in CRF between the two groups before chemotherapy ( $\mathrm{P}>0.05)$, and the total fatigue during and after chemotherapy was lower in the observation group than in the control group $(\mathrm{P}<0.05)$ (Table 1).

\section{Comparison of SF-36 scores between the two groups of patients on lifestyle therapy}

The post-intervention SF-36 Brief Health Score was statistically significantly higher in the observation group than in the control group $(\mathrm{P}<0.05)$ (Table 2$)$.

\section{Comparison of the psychological status of the two groups of patients}

There was no difference in psychological status between the two groups before the intervention $(\mathrm{P}>0.05)$; after the intervention, patients in the observation group had a better psychological status than those in the control group $(\mathrm{P}<0.05)$ (Table 3).

\section{Comparison of anxiety and depression in two groups of patients after 2nd, 4th, and 6th time}

The anxiety and depression scores of the two groups after the second chemotherapy were not statistically significant $(\mathrm{P}>0.05)$, and the anxiety and depression scores of the observation group after the fourth and sixth chemotherapy were lower than those after the second, and lower than those of the control group $(\mathrm{P}<0.05)$ (Table 4$)$.

Table 1. Comparison of CRF before, during, and after chemotherapy in two groups of patients $(\bar{x} \pm s)$

\begin{tabular}{ccccc}
\hline Group & physical fatigue & emotional fatigue & cognitive fatigue & Total fatigue \\
\hline Before chemotherapy in the observation group $(\mathrm{n}=45)$ & $3.31 \pm 1.36$ & $3.69 \pm 1.76$ & $4.18 \pm 1.53$ & $11.28 \pm 4.14$ \\
Before chemotherapy in the control group $(\mathrm{n}=38)$ & $3.57 \pm 1.18$ & $4.18 \pm 1.58$ & $4.41 \pm 1.95$ & $12.76 \pm 4.63$ \\
$\mathrm{t}$ & 1.140 & 0.845 & 0.347 & 0.723 \\
$\mathrm{P}$ & 0.265 & 0.431 & 0.723 & 0.462 \\
Observation group in chemotherapy $(\mathrm{n}=45)$ & $11.38 \pm 1.92$ & $4.37 \pm 3.58$ & $6.45 \pm 1.37$ & $24.24 \pm 7.23$ \\
Control group in chemotherapy $(\mathrm{n}=38)$ & $12.97 \pm 1.53$ & $5.95 \pm 4.69$ & $7.69 \pm 1.95$ & $27.87 \pm 8.38$ \\
$\mathrm{t}$ & 4.269 & 2.029 & 2.276 & 2.327 \\
$\mathrm{P}$ & $<0.001$ & 0.044 & 0.025 & 0.024 \\
After chemotherapy in the observation group $(\mathrm{n}=45)$ & $4.48 \pm 1.69$ & $5.53 \pm 2.53$ & $5.78 \pm 1.96$ & $16.39 \pm 6.33$ \\
After chemotherapy in the control group $(\mathrm{n}=38)$ & $5.39 \pm 1.74$ & $6.79 \pm 2.25$ & $6.97 \pm 1.81$ & $18.84 \pm 5.65$ \\
$\mathrm{t}$ & 2.023 & 2.375 & 2.275 & 2.249 \\
$\mathrm{P}$ & 0.047 & 0.023 & 0.026 & 0.026 \\
\hline
\end{tabular}


Table 2. Comparison of SF-36 quality of life scores between two groups of patients $(\bar{x} \pm s)$

\begin{tabular}{|c|c|c|c|c|c|c|c|c|c|}
\hline Group & $\mathrm{n}$ & $\begin{array}{l}\text { Mental } \\
\text { Health }\end{array}$ & $\begin{array}{l}\text { Emotional } \\
\text { Functions }\end{array}$ & $\begin{array}{c}\text { Social } \\
\text { Functions }\end{array}$ & Vitality & $\begin{array}{l}\text { General } \\
\text { Health }\end{array}$ & $\begin{array}{c}\text { physical } \\
\text { health }\end{array}$ & $\begin{array}{l}\text { physical } \\
\text { functions }\end{array}$ & $\begin{array}{c}\text { Physiological } \\
\text { Functions }\end{array}$ \\
\hline $\begin{array}{l}\text { Before obser- } \\
\text { vation group } \\
\text { intervention }\end{array}$ & 45 & $70.45 \pm 3.98$ & $82.52 \pm 6.45$ & $78.45 \pm 7.21$ & $79.65 \pm 18.77$ & $80.75 \pm 3.42$ & $79.62 \pm 3.98$ & $70.28 \pm 5.34$ & $67.26 \pm 3.55$ \\
\hline $\begin{array}{l}\text { After observa- } \\
\text { tion group } \\
\text { intervention }\end{array}$ & 45 & $79.43 \pm 14.81$ & $85.76 \pm 6.52$ & $85.54 \pm 6.45$ & $87.28 \pm 20.61$ & $83.72 \pm 16.71$ & $83.51 \pm 6.91$ & $79.87 \pm 8.24$ & $79.76 \pm 6.34$ \\
\hline $\begin{array}{l}\text { Before Control } \\
\text { Group Inter- } \\
\text { vention }\end{array}$ & 38 & $50.78 \pm 11.36$ & $45.97 \pm 5.79$ & $54.92 \pm 13.31$ & $48.21 \pm 18.52$ & $57.14 \pm 13.96$ & $72.51 \pm 4.43$ & $70.92 \pm 5.72$ & $70.62 \pm 3.23$ \\
\hline $\begin{array}{l}\text { After Control } \\
\text { Group Inter- } \\
\text { vention }\end{array}$ & 38 & $62.89 \pm 15.64$ & $52.25 \pm 7.48$ & $64.93 \pm 14.55$ & $56.48 \pm 19.40$ & $67.74 \pm 15.94$ & $77.57 \pm 5.35$ & $75.72 \pm 7.34$ & $75.54 \pm 5.71$ \\
\hline $\begin{array}{l}\mathrm{t} \\
\mathrm{P}\end{array}$ & & $\begin{array}{l}5.392 \\
0.001\end{array}$ & $\begin{array}{c}23.462 \\
0.001\end{array}$ & $\begin{array}{l}4.395 \\
0.001\end{array}$ & $\begin{array}{l}4.467 \\
0.001\end{array}$ & $\begin{array}{l}4.891 \\
0.001\end{array}$ & $\begin{array}{l}4.794 \\
0.001\end{array}$ & $\begin{array}{l}4.598 \\
0.001\end{array}$ & $\begin{array}{l}5.582 \\
0.001\end{array}$ \\
\hline
\end{tabular}

Table 3. Comparison of the psychological status of two groups of patients after the intervention with the palliative approach $(\bar{x} \pm s)$

\begin{tabular}{cccccc}
\hline group & $\mathrm{n}$ & $\begin{array}{c}\text { Social Behavioral } \\
\text { Issues }\end{array}$ & $\begin{array}{c}\text { Personal Emotional } \\
\text { Issues }\end{array}$ & $\begin{array}{c}\text { Habits } \\
\text { Issues }\end{array}$ & $\begin{array}{c}\text { Study Work } \\
\text { Issues }\end{array}$ \\
\hline Before observation group Intervention & 45 & $3.71 \pm 0.86$ & $6.57 \pm 0.39$ & $1.83 \pm 0.41$ & $1.51 \pm 0.24$ \\
After observation group Intervention & 45 & $2.17 \pm 0.14$ & $3.13 \pm 0.27$ & $0.87 \pm 0.31$ & $0.57 \pm 0.31$ \\
Before Control Group Intervention & 38 & $3.47 \pm 0.42$ & $6.41 \pm 0.24$ & $1.84 \pm 0.16$ & $1.47 \pm 0.28$ \\
After Control Group Intervention & 38 & $2.24 \pm 0.32$ & $4.11 \pm 0.41$ & $1.07 \pm 0.12$ & $1.13 \pm 0.26$ \\
$\mathrm{~T}$ & & 14.572 & 17.896 & 3.864 & 14.429 \\
$\mathrm{P}$ & & 0.001 & 0.001 & 0.001 & 0.001 \\
\hline
\end{tabular}

Table 4. Comparison of anxiety and depression after the second, fourth, and sixth chemotherapy treatments in the two groups $(\bar{x} \pm s)$

\begin{tabular}{ccccc}
\hline Group & $\mathrm{n}$ & the second & the fourth & the sixth \\
\hline Observation Group Anxiety Score & 45 & $41.27 \pm 3.58$ & $40.78 \pm 6.74$ & $36.72 \pm 6.44$ \\
Control group anxiety score & 38 & $43.64 \pm 3.17$ & $44.53 \pm 5.78$ & $43.53 \pm 5.57$ \\
$\mathrm{t}$ & & 0.548 & 2.306 & 2.159 \\
$\mathrm{P}$ & & 0.615 & 0.025 & 0.031 \\
observation group Depression score & 45 & $49.58 \pm 8.32$ & $45.73 \pm 4.54$ & $41.18 \pm 6.54$ \\
Control Group Depression Score & 38 & $48.79 \pm 8.45$ & $51.53 \pm 5.32$ & $49.78 \pm 5.78$ \\
$\mathrm{t}$ & & 0.124 & 3.883 & 5.689 \\
$\mathrm{P}$ & & 0.923 & 0.001 & 0.001 \\
\hline
\end{tabular}

\section{Discussion}

The main treatment for cervical cancer is comprehensive treatment, and prolonged comprehensive treatment will induce fatigue symptoms with regularity, causing patients to suffer from anxiety, depression and other negative emotions, which will affect the treatment effect and quality of life of patients. According to the study [8], before chemotherapy, cancer patients showed a low level of fatigue, and after chemotherapy, due to the side effects of chemotherapy, patients showed severe fatigue symptoms and sustained moderate fatigue in emotion and cognition. The results of this study showed that there was no statistical difference in CRF between the two groups before chemotherapy, and the total fatigue during and after chemotherapy in the observation group was lower than that in the control group $(\mathrm{P}<0.05)$, which was similar to the conclusion of ZHANG [9]. Bennett [10] pointed out that the cognitive and emotional dimensions of fatigue of cervical cancer patients treated with combination therapy were significantly higher than that of cervical cancer patients treated with radiotherapy alone, and the cognitive and emo- 
tional dimensions of fatigue were significantly higher than that of cervical cancer patients treated with radiotherapy alone in the treatment session. Cognitive fatigue was most pronounced during chemotherapy, presumably because patients treated with combination therapy were often in the advanced stages of cervical cancer.

When cervical cancer patients experience symptoms of CRF, the common self-relieving methods include sleep, napping, and light diet. From an overall analysis, patients' perceptions suggest that sleep and reduced activity can alleviate fatigue, which is a common way for patients to relieve fatigue, similar to the findings of Ebede [11]. In the case of Chinese patients, the traditional perception is that patients need to stay in bed for a long period of time, and Zhang [12] found that bed rest is less effective in relieving fatigue, and that CRF can be relieved more effectively with the help of certain exercise guidance and intervention [13].

This study adopted yoga resistance training and psychological intervention as a way to alleviate CRF. Yoga training includes meditation, relaxation, imagination, breathing adjustment, stretching and physical exercise, which can meet the physical exercise needs of cervical cancer patients and is one of the safe aerobic exercises. It can improve patients' stiffness, relieve tension, and help improve the fatigue level of cervical cancer chemotherapy patients. The results of this study showed that the SF-36 brief quality of life score of patients in the observation group was better than that of the control group after the intervention, and the difference was statistically significant $(\mathrm{P}<0.05)$, and the CRF of patients was significantly improved after the intervention, and the quality of life was significantly improved. After the combined psychological intervention, the psychological health of the intervention group was better than that of the control group. On the one hand, psychological intervention can relieve the negative emotions of cervical cancer patients, on the other hand, it can also enhance the patients' tolerance to chemotherapy and improve the toxic side effects of drugs. The combination of yoga and psychological intervention had a positive effect on the psychological, physiological and spiritual state of cervical cancer chemotherapy patients.

Studies have reported [14] that yoga can improve the quality of life of cancer patients and improve their adverse psychological reactions such as anxiety and tension. The results of this study showed that the anxiety and depression scores of the observation group after the 4th and 6th chemotherapy were lower than those of the 2nd chemotherapy and lower than those of the control group $(\mathrm{P}<0.05)$. The group yoga exercise can promote communication between patients, increase patients' confidence to overcome the disease, and improve anxiety and depression. The results of this study showed that the quality of life of the two groups of patients improved by $85.71 \%$ and $66.67 \%$ respectively, and the quality of life of the observation group was significantly higher than that of the control group. After the yoga exercise and psychological intervention, the physical and psychological health of the observation group was significantly improved compared to the control group.

The physical, emotional and cognitive fatigue of cervical cancer chemotherapy patients made their quality of life poorer, accompanied by anxiety, depression and other negative emotions. Yog a and psychological intervention can effectively improve the overall fatigue of cervical cancer chemotherapy patients and improve their quality of life. Since the intervention period in this study was only during the chemotherapy period, which was relatively short, and the patients were not observed during the rehabilitation period, later studies can observe the effect of yoga exercise and psychological intervention on patients' CRF during the rehabilitation period of cervical cancer.

\section{Declaration}

All authors of this paper declare they have no conflicts of interest.

\section{Acknowledgements}

This study was approved by the Affiliated Hospital of Shaanxi University of Traditional Chinese Medicine (2020HL006).

\section{References}

[1] Retz, M. and Karl, A. (2018). Bladder cancer: Current diagnosis and treatment modalities [J]. Urologe A, 57(6): 655-656.

[2] Arring, N. M., Barton, D. L., Brooks, T., et al. (2019). Integrative Therapies for Cancer-Related Fatigue [J]. Cancer J., 25(5): 349-356.

[3] Mohandas, H., Jaganathan, S. K., Mani, M. P., et al. (2017). Cancer-related fatigue treatment: An overview [J]. J Cancer Res Ther., 13(6): 916-929.

[4] Georden, J., M. Gollish, G. Trudel, N. Rutkowski, et al. (2020). A perfect storm and patient-provider breakdown in communication: two mechanisms underlying practice gaps in cancer-related fatigue guidelines implementation [J]. Supportive Care in Cancer, 2020 (prepublish). 
[5] Ye, H. R., Xu, S. D., Li, G. D., et al. (2019). Reliability and validity analysis of the SF-36 Concise Quality of Life Scale in patients with chronic prostatitis [J]. China Health Care Management, 36(01): 66-68.

[6] Xu, Y. L., Fu, D. Y. (2018). Correlation between spousal psychological resilience and anxiety-depressive state and coping style in young and middle-aged breast cancer patients [J]. Chinese Journal of Surgical Oncology, 10(05): 337-340.

[7] Lerdal, A., Kottorp, A., Gay, C., et al. (2016). A Rasch Analysis of Assessments of Morning and Evening Fatigue in Oncology Patients Using the Lee Fatigue Scale [J]. J Pain Symptom Manage, 51(6): 1002-1012.

[8] Ebede, C. C., Jang, Y., Escalante, C. P. (2017). Cancer-Related Fatigue in Cancer Survivorship [J]. Med Clin North Am., 101(6): 1085-1097.

[9] Zhang, Q., Li, F., Zhang, H., et al. (2018). Effects of nurse-led home-based exercise \& cognitive behavioral therapy on reducing cancer-related fatigue in patients with ovarian cancer during and after chemotherapy: A randomized controlled trial [J]. Int J Nurs Stud., 78: 52-60.

[10] Bennett, S., Pigott, A., Beller, E. M., et al. (2016). Educational interventions for the management of cancer-related fatigue in adults [J]. Cochrane Database Syst Rev., 11: D8144.

[11] Ebede, C. C., Jang, Y., Escalante, C. P. (2017). Cancer-Related Fatigue in Cancer Survivorship [J]. Med Clin North Am., 2017, 101(6): 1085-1097.

[12] Zhang, Y., Lin, L., Li, H., et al. (2018). Effects of acupuncture on cancer-related fatigue: a meta-analysis [J]. Support Care Cancer, 26(2): 415-425.

[13] Van Vulpen, J. K., Peeters, P. H., Velthuis, M. J., et al. (2016). Effects of physical exercise during adjuvant breast cancer treatment on physical and psychosocial dimensions of cancer-related fatigue: A meta-analysis [J]. Maturitas, 85: $104-111$.

[14] Jong, M. C., Boers, I., Schouten, V. D. V. A., et al. (2018). A Randomized Study of Yoga for Fatigue and Quality of Life in Women with Breast Cancer Undergoing (Neo) Adjuvant Chemotherapy [J]. J Altern Complement Med., 24(9-10): 942-953. 